\title{
The Venerable Subclavian Line
}

\author{
Nicholas Teman MD, Charles Hobson MD, Reid Tribble MD, Curt Tribble MD \\ Division of Cardiac Surgery, University of Virginia, Charlottesville, VA, USA
}

\section{INTRODUCTION}

Risk comes from not knowing what you're doing.

-Warren Buffett

In this treatise, we will address one of the higher-risk procedures, subclavian vein cannulation, that a practitioner may undertake in the care of complex patients. All cardiothoracic surgeons and their trainees will need, on occasion, to put in central lines in a variety of circumstances, including in the operating room, in the intensive care unit, in emergency circumstances, and, occasionally, when other practitioners have been unsuccessful in their attempts to place a central line.

We will describe, in detail, the anatomy of the subclavian vein, the preparation of the patient for subclavian vein cannulation, the infraclavicular approach to cannulation of the vein, and a few notes about the supraclavicular approach to the subclavian vein. It is self-evident that the priorities of central venous cannulation include safety of insertion, minimizing clot formation, and avoiding infection. We will dwell primarily on the principles of safe subclavian line insertion.

\section{After the code, a perfusing rbythm \\ back and a new \\ chest tube to suction, my chief offered \\ some feedback on \\ my central line: \\ the needle was \\ in the wrong place, just like me.}

-An Intern's Recollection of a Night at the VA, July 2004

Doug Hester, MD [Hester, 2004]

\section{THE HISTORY OF CENTRAL VENOUS ACCESS}

The earliest development of central venous cannulation was prompted by the initial development of parenteral alimentation with hypertonic solutions, led most prominently by Jonathon Rhoads and Stanley Dudrick at the University

Correspondence: Curt Tribble, MD, Professor of Cardiothoracic Surgery, University of Virginia Health System, Division of Cardiac Surgery, Box 800679, Charlottesville, VA 22908; 434-243-9250 (e-mail: ctribble@virginia.edu). of Pennsylvania in the 1960's. [Dudrick, 1969. Dudrick, 2003] One of our colleagues, R. Scott Jones, was a co-chief resident at Penn, and he has regaled us with tales of those early days of intravenous nutrition research. Once these strategies for intravenous alimentation had been shown to be successful and became more widely used, techniques for reliable access to the central venous system were developed. Many of the early reports of central venous access described the use of the supraclavicular approach to the subclavian vein. [Patrick, 2009] Gradually, the infraclavicular approach became more common. In this treatise we will focus primarily on the infraclavicular approach, while adding a few additional notes about the supraclavicular approach. More recently, both internal jugular (IJ) venous catheters and peripherally inserted central catheters (PICC), often placed using ultrasound guidance, have come into widespread use. However, these catheters have their own disadvantages and are, therefore, not always the optimal strategies for central venous access. Even with ultrasound guidance, the placement of an IJ catheter has a risk of both carotid artery injury and pneumothorax. PICC lines have a higher incidence of thrombosis compared to other central lines and they are both longer and have smaller diameter channels which makes them less effective for the rapid infusion of blood or colloid. [Honore, 2021]

\section{THE ANATOMY OF THE SUBCLAVIAN VEIN}

\section{Anatomy is destiny. \\ —Sigmund Freud}

There is no doubt that all who carry out procedures, whether surgical or otherwise, must have a complete understanding and mastery of the anatomy involved. One must understand the anatomy pertinent to any procedure and be able to visualize that anatomy accurately in order to carry out that procedure. These principles are true for most procedures, and they are vital to accessing the subclavian vein safely and successfully. Though the saying that 'anatomy is destiny' comes from the realm of psychology, it is certainly pertinent to the realm of surgical procedures. [Tubbs, 2021] Thus, we will describe in detail the anatomy pertinent to cannulating the subclavian vein.

The axillary vein begins at the lower border of the teres major muscle as a continuation of the brachial vein, which runs in the delto-pectoral groove. It traverses the axilla as it moves towards the middle third of the clavicle. As the axillary vein crosses the lateral border of the first rib, it becomes the 
subclavian vein. The subclavian vein rises behind the clavicle and arches over the first rib anterior to the insertion of the anterior scalene muscle onto the first rib. The vein reaches its most cephalad position at this point, as it crosses over the first rib. The anterior scalene muscle separates the subclavian vein from the subclavian artery at that point. Just beyond the medial edge of the anterior scalene muscle, the subclavian vein joins the internal jugular vein to form the brachiocephalic vein.

The subclavian vein is bordered anteriorly by the clavicle, the subclavius muscle, and the costoclavicular ligament. The vein is bordered inferiorly by the first rib, while, lateral to the first rib, the vein passes over the uppermost part of the pleura of the lung. In the setting of hyperinflation of the lung, as occurs in emphysema, the subclavian vein can be elevated above its normal position. This type of elevation of the apex of the lung may also be present in patients on a ventilator, of course. And, in patients with malnutrition or emphysema, the lung can be even closer to the expected trajectory of a needle being aimed at the subclavian vein than would be the case in a normal person. The anterior scalene muscle is generally about $1 \mathrm{~cm}$ in diameter at its insertion on the first rib, and in a malnourished person, this muscle may be even smaller, which means that the artery and vein may be even closer to each other in such patients.

More medially, the internal mammary artery, a branch of the subclavian artery, passes behind the first rib and the subclavian vein as it descends into the chest, eventually lying posterior to the ribs of the anterior chest wall. The thoracic duct, after crossing in front of the left anterior scalene muscle, enters the junction of the left subclavian vein and the left internal jugular vein. The following figures shows the relationship of the right subclavian vein to the clavicle and first rib, and to the anterior scalene muscle, which separates the subclavian vein from the subclavian artery:
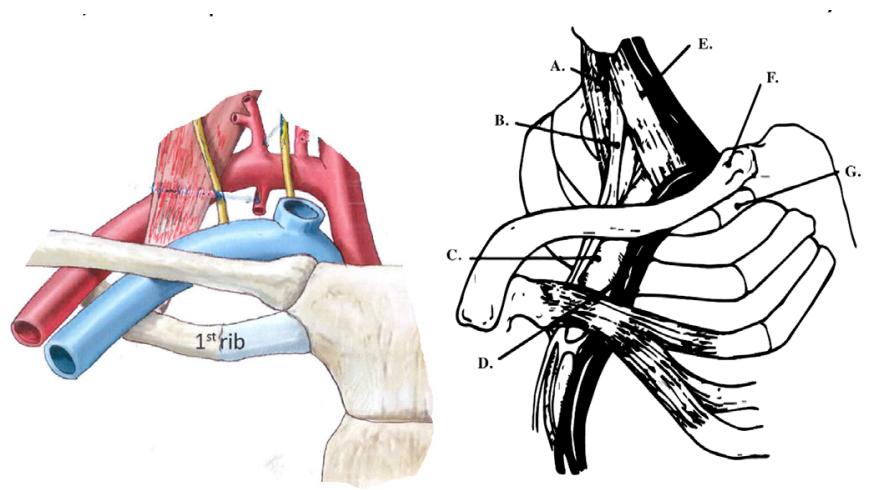

A: middle scalene muscle. B: brachial plexus. C: subclavian artery. D: subclavian vein. E: anterior scalene muscle. F: clavicle. G: first rib.

The next figure shows the external landmarks of the deltopectoral groove, which is located between the delto-humeral prominence and the pectoralis major muscle:

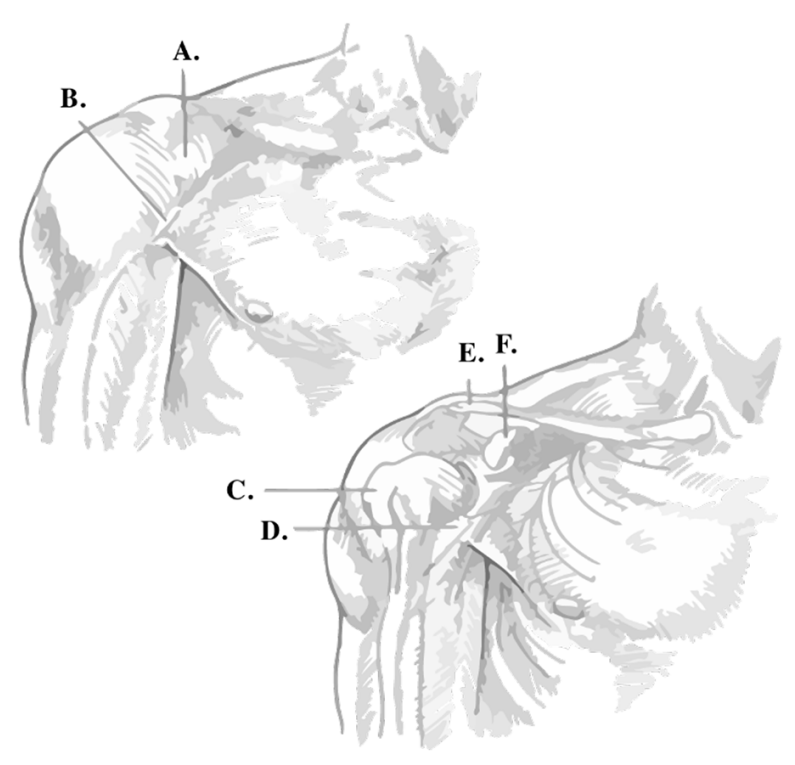

(A: coracoid prominence. B: deltopectoral groove. C: head of humerus. D: cephalic vein. E: clavicle. F: coracoid process.

The following figure demonstrates how one can feel the somewhat bulky anterior scalene muscle posterior to the thinner sternocleidomastoid muscle. The anterior scalene represents most of the muscle mass palpable in the anterior aspect of this region of the neck. Remember that the anterior scalene muscle passes posterior to the clavicle and inserts on the first rib. In this picture, the muscle edge anterior and medial to the examiner's thumb is the sternocleidomastoid, while the palmar surface of the tip of the examiner's thumb lies on the anterior scalene muscle:

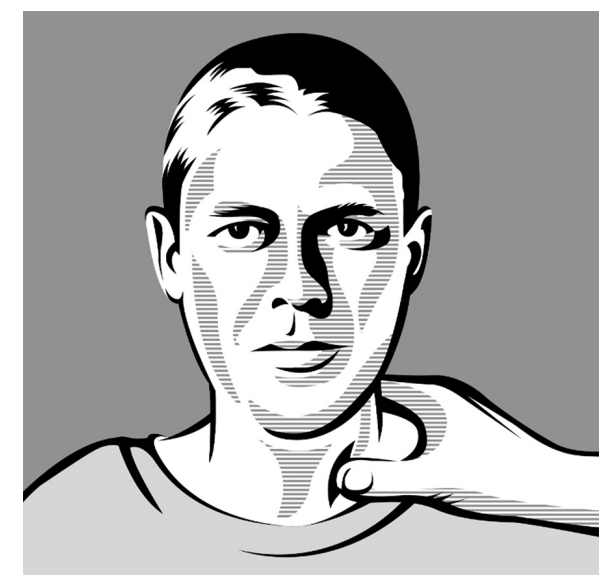

More specifically, when you place your thumb beside the lateral edge of the sternocleidomastoid muscle (SCM), the palmar surface of the distal phalanx of your thumb will rest on the anterior surface of the anterior scalene muscle, which is a few centimeters posterior to the SCM which inserts on the superior aspect of the sternum. The subclavian vein will, 
therefore, lie just below (inferior to) your thumb, running as the vein does behind the sternum and superior to the first rib, onto which the anterior scalene inserts. Understanding the anatomy of these landmarks will allow you to visualize in three dimensions where you will want your needle to enter the subclavian vein. Thus, the precise spot you will want to aim for is a cylinder about the size of the distal phalanx of your thumb.

I skate to where the puck is going to be, not where it has been. -Wayne Gretsky [Brainy Quote]

Once you are able to visualize the anatomy, you will know precisely where your needle needs to go. The cylindrical target zone is behind the clavicle, above the first rib, and

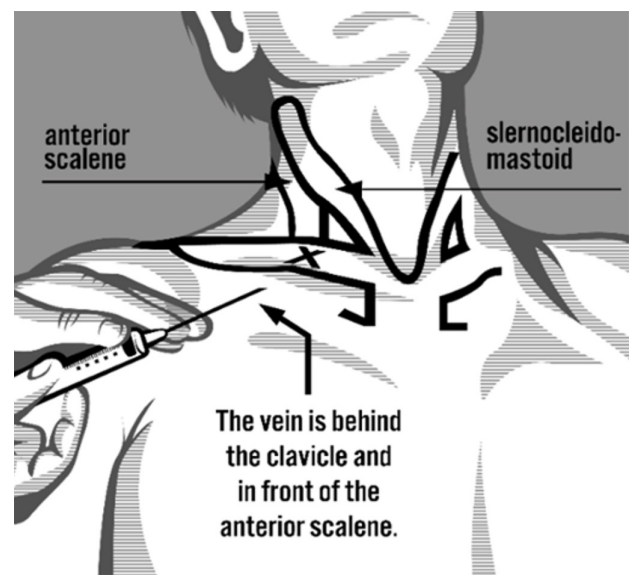

anterior to the anterior scalene muscle, posterior to the site marked with the ' $\mathrm{X}$ ' in this figure:

Though not the primary focus of this treatise, the vein can also be cannulated safely from above the clavicle. The supraclavicular approach is, most certainly an option in certain situations. [Patrick, 2009] However, the following figure is presented here primarily to reinforce how one can and should visualize the 'box' or cylinder where the needle should enter the vein, either from below or from above the clavicle:

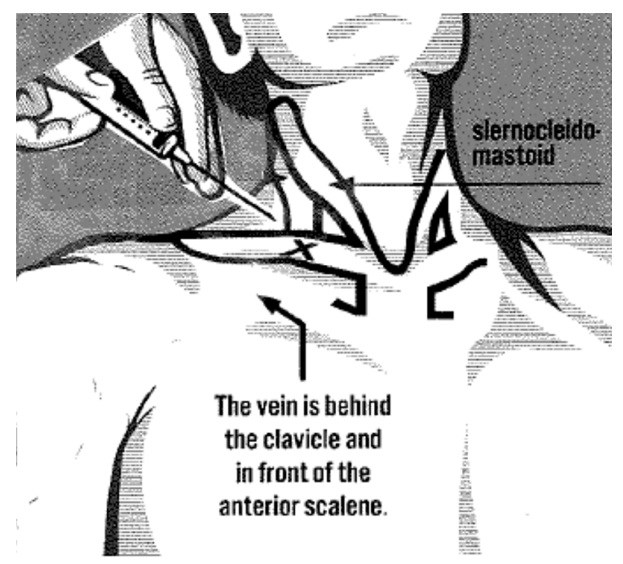

As noted earlier, the supraclavicular approach was actually adopted prior to the subclavian approach becoming the more common method of subclavian vein line placement. This earlier strategy may have been influenced by the fact that pediatricians had long known this approach to be a reliable and relatively safe way to draw blood from a child. The supraclavicular approach to the subclavian vein likely became less common when total parenteral nutrition came into widespread use in the late 1970's and dressing of the line insertion site to prevent infection was considerably easier and more reliable when that site was on the upper chest rather than in the neck. However, the supraclavicular approach can be a better approach to the subclavian vein in very heavy people, in children, or, perhaps, in patients with hyper-expanded lungs. Some practitioners also believe that this approach is safer in urgent or emergent situations. [Tomar, 2013] However, because dressing these lines when the insertion site is in the neck is challenging, the supraclavicular approach is likely best reserved for patients who are relatively immobile, such as those in an intensive care unit.

\section{OBTAINING CONSENT FOR CENTRAL LINE PLACEMENT}

The first step in preparing the patient for any procedure is to explain the procedure to the patient and / or the patient's family and to obtain informed consent. [Tribble, 2019] Potential complications should be explained, of course. Complications that may occur include: pneumothorax, injury to the subclavian artery, injury to the thoracic duct (on the left), hemothorax, and entry of air into the right ventricle. Later complications include infection, thrombosis of the vein, or clot formation at the tip of the catheter. The patient, if awake, should be told that there may be some discomfort which can occur if the needle touches the periosteum of the clavicle, which is quite sensitive.

\section{MARKING THE SITE}

We have long thought that marking the intended sites of incisions (or insertions, as in the case of line placement) is an important part of every procedure. While this practice may be eschewed by experienced practitioners in some settings, there is no doubt that the value of marking the anatomy is greater when less experienced proceduralists are involved. Not only can one outline the pertinent anatomy for purposes of visualization, but this practice can also assure a supervisor that a less experienced protégé understands the anatomy and the proper approach. As an aside, we are not necessarily suggesting that the extensive markings shown in the illustrations are necessary.

\section{VISION: THINK LIKE A SCULPTOR}

The person inserting the needle must be capable of thinking in three dimensions, visualizing the precise site that the needle should enter the subclavian vein. It has been our 
observation that, far too often, the practitioner, especially if inexperienced, will attempt to insert the needle in a rote manner, without visualizing the target properly. As Eliyahu Goldratt writes in his book, It's Not Luck, savvy practitioners of any type must have a clear vision of what they hope to accomplish. [Goldratt, 1994]

Can you imagine a sculptor chiseling away at a block of stone not having a vision of what he or she is trying to create? These same principles apply in almost all procedures, from line insertion to most surgical operations. [Tribble, 2020 \& 2021]

\section{GATHERING THE NECESSARY SUPPLIES FOR CENTRAL LINE INSERTION}

All the appropriate supplies needed to insert the catheter under strict sterile conditions should be collected and organized. In fact, there is usually a single standardized central line kit for any given institution. There are also drape kits that are often called "universal barrier sets." These kits should contain an extra-large sterile gown, as well as a cap, surgical masks, prep solution, a full body drape (ideally with a work hole surrounded by adhesive so that a sterile work area is easily maintained), Benzoin or sticky skin prep pads, and a high quality line dressing. These central line kits are available in virtually all areas where central lines will be placed and should contain all the necessary materials for the procedure itself, including local anesthetic, needles, wires, and catheters. Ultrasound is not particularly useful for placement of subclavian lines, in contrast to its proven usefulness for internal jugular vein cannulation, though some practitioners have touted the usefulness of using a Doppler to ensure the patency of the subclavian vein prior to starting the procedure.

You should also round up extra towels (with cloth towels being preferred because they lie more solidly on and conform better to the patient than do paper towels). And, it is worth considering having some extra sheets available, which should allow you to cover the patient from head to toe, with the sterile sheets extending widely to either side of the patient, once placed. Keep in mind that central line procedures require the use of a guidewire, the 'back side' of which may end up lying, at some point during the insertion, quite a ways to the side of the patient.

It is best to use a 10cc, non-Luer lock (also called a 'slip tip') syringe because they are much easier to disconnect from the needle than are Luer lock syringes. A larger syringe is not only unnecessary, but it may also be counterproductive, for several reasons, including being too bulky for the adjacent anatomy, perhaps creating more suction than is optimal, and being harder to hold properly in your hand.

It is also a good idea to round up pair of scissors, which can be useful for tailoring the drapes and for cutting the anchoring sutures at the end of the procedure. One should also round up some suture of one's choice for anchoring the line. Silk sutures (2-0 or 0$)$ are good choices, though 3-0 monofilament sutures are preferred by some practitioners. Having extra suture handy is also a good idea, in case you need an extra one for some reason.
Another subtle but important issue to consider when gathering your supplies is that a longer catheter may be preferable if the left subclavian vein is to be cannulated, as the trajectory from insertion to the junction of the superior vena cava and the right atrium is slightly longer from the left side than from the right. A 15 centimeter catheter is appropriate for a line placed from the right side, while an 18 to 20 centimeter catheter may be more appropriate for a line placed from the left.

\section{CREATING YOUR WORKSPACE}

It is very helpful to have a rolling bedside table, properly draped to create a 'workspace,' on which you can lay out the supplies that will be needed so that the gear is both organized and near the operator, as well as not having those supplies sitting on the patient, who may move a bit during the procedure. Each component of the kits should be evaluated for its usefulness and prepared for use. You should strive to remove anything unnecessary from the field, to reduce clutter and distractions. You must flush the lumen or lumens of the line you intend to use. Throw away all wrappers or containers to minimize clutter. Know where all of the supplies that you intend to use are located.

\section{POSITIONING THE PATIENT}

The patient should be properly positioned, once the necessary preparations are complete, usually slightly closer to the side of the bed on the side that you intend to cannulate, especially if the bed is wide, as are many ICU beds. Most practitioners place a rolled towel or sheet between the patient's shoulder blades. The benefit of doing this is that the shoulder will usually drop backwards a bit. The potential disadvantage of this strategy is that the vein may be a bit more compressed by the clavicle by this maneuver. Having the shoulder moved a bit superiorly (like a shrug) can create a little more space under the clavicle. The patient's head should be turned slightly away from the side to be cannulated. The following diagrams illustrate the desired position of the shoulder, which allows the deltoid muscle and head of the humerus to drop posteriorly and which will allow optimal orientation of the syringe and needle, as the needle is being inserted, which should be parallel to the floor.
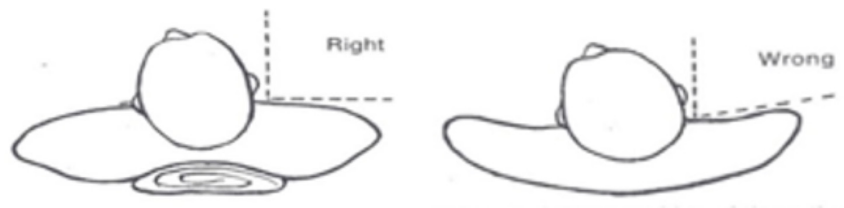

To fill the vein as much as feasible, the patient should be placed in the Trendelenburg position, with the head of the bed slightly down $\left(\sim 10^{\circ}\right)$. Furthermore, if there is any concern about the patient being hypovolemic, it can be useful to have 
some crystalloid solution infusing just prior to and during the procedure to ensure that the venous system is full. One can often see the external jugular vein distend when the head is lowered, which, if visible, is a reliable way to assess how much to tilt the bed. This position is said to also lessen the chance of air being sucked into the venous system and the right side of the heart, which can occur when an open or uncovered needle is in the vein and the patient breathes in.

It is worth noting that it is optimal if an assistant can position themselves at the head of the bed in order to talk to the patient and to keep the drapes from lying directly on the patient's face, especially if the patient is awake. Both this 'support' person and the person doing the procedure should talk to the patient, not only to explain what is happening at each step but to also comfort the patient. Remember that a calm human voice is an anesthetic as well as a mild sedative. Some have suggested placing a nasal cannula with oxygen flowing through it near the patient's face, blowing away from the side you are working on, to provide a cooling 'breeze' to the patient while their head is at least somewhat covered during the procedure. One can also consider placing soft restraints on the wrists, as you do not want the patient to inadvertently reach up at an inopportune moment and disrupt the process.

\section{PREPPING THE SKIN OF THE PATIENT}

\section{It's better to prep widely than to wish that you had.} -Dan Waters

The skin is prepared with chlorhexidine solution. Some prefer to clean the area with alcohol first in order to remove skin oils. Some practitioners also like to apply a first layer of prep solution early, prior to the 'final' prep, as this layer would be in place longer, doing its work, in a time dependent fashion, of killing micro-organisms on the skin. An old adage about the prepping of a patient for a procedure is that one can never prep too widely. Specifically, prep a much larger area than you might imagine needing for the procedure. This strategy keeps unprepped skin from 'working its way' into the sterile field. It also allows wide draping so that the landmarks will remain visible. The prepped area should include the edge of the jaw, the neck up to the ear, the neck and chest wall to the other side of the midline, the anterior chest to the nipple, the lateral chest well past the anterior axillary line, and the upper shoulder. Masks and caps should be donned by all involved in the procedure, prior to the application of the chlorhexidine.

\section{PREPPING THE PRACTITIONER}

The person doing the procedure should have already donned a cap and mask, prior to prepping the patient. Then that person must wash his or her hands and then put on a sterile gown. The hands should NOT extend out beyond the cuffs until gloves are donned. You must to know how to glove yourself in a sterile manner. [glove donning video] If this strategy is not followed, the cuffs of both the gown and the gloves will not remain sterile. The key to gloving yourself is to lay the gloves palm to palm with the fingers facing towards you and to then fold the glove over your fingers. It is also preferable to double glove, for several reasons. First of all, double gloving has been shown to not reduce tactile sensation, and many, if not most, surgeons double glove for all procedures. [Mischke, 2014] Among the advantages of double gloving is that if you have any sense that you've touched something unsterile, it is really easy to exchange your outer gloves for a new set of gloves.

\section{DRAPING THE PATIENT}

The first step in draping the patient is to place sterile towels around the site, optimally with cloth towels. The very first towel placed should be one that is unfolded, 'wadded up,' and placed between the neck and the shoulder. The next towel should be placed on the chest, well below the intended insertion site. The remaining two or three towels should then be placed to completely encircle the field, while placing them widely enough apart to allow continued visualization and palpation of the external anatomical landmarks. Towels lie optimally when folded on their long edge prior to placing them. Adhesive Ioban drapes placed over the site are unnecessary and may cause trouble by 'binding' on the needles, wires, and catheters during the procedure and by endangering the newly inserted catheter when pulling the sticky drape off after the procedure is completed. The large sheets or drapes are then placed to create a very wide sterile field. They should be placed in such a way that they do not lie directly on top of an awake patient's face. One way to accomplish that goal is to slide a Mayo tray in from the opposite side of the bed so that the sheets can be held away from the patient's face. It is at this point that someone on the team should be ready to sit on a stool beside the patient's head so that the patient can be talked to and comforted if they are awake. If sheets with holes already in them are used, the hole can, and usually should, be enlarged with scissors to maintain a proper sterile field and to maintain your ability to see the pertinent landmarks. Never use a knife when 'tailoring' the sheets. Using a knife for this purpose is awkward at best and may be dangerous for the patient and practitioner. Scissors should be used for this purpose in all cases.

After draping, one should take time to identify the landmarks again. Particular attention should again be paid to the location of the anterior scalene muscle. It is worth reminding yourself that the target on the subclavian vein is a postage stamp-sized 'box' or cylinder behind the clavicle, in front of the anterior scalene muscle, and on top of the first rib. These landmarks can be remarked, if necessary, after prepping and prior to inserting the needle.

\section{THE CHECKLIST}

Prior to starting the actual procedure, you and your team must run through a pre-procedure checklist. [Calland, 2002] A printed check list should be used. The issues to be reviewed 
include acknowledging that the following have been appropriately accomplished: confirmation of the patient's identity, the procedure to be performed and the side to be accessed, assurance that informed consent has been obtained (or that there is documentation in the chart that the procedure is urgent and that consent could not be obtained), proper hand washing, proper sterile attire for the proceduralist and assistant, proper preparation of the insertion site, proper positioning of the patient, proper draping, and that all the necessary supplies are on hand.

\section{SELECTING THE SITE FOR NEEDLE INSERTION}

With the target site visualized in 'your mind's eye,' the site for the initial needle stick is selected. You must have 'a sense of where you are' and a sense of where you are aiming. [McPhee, 1999] The initial puncture site in the skin should allow a slightly cephalad trajectory of the needle toward the target site on the vein. This puncture site will usually be slightly lateral to the mid-clavicular line, in the delto-pectoral 'depression'. Sometimes a slightly more medial site for the initial puncture will be required if the shoulder cannot be adequately positioned posteriorly. The goal is to have the needle travel straight towards the expected site of entrance into the vein, behind the clavicle, on top of the rib, and in front of the anterior scalene muscle. This trajectory should be nearly horizontal (that is, parallel to the floor), with a slight cephalad trajectory, as noted earlier. The initial entry site should not be so medial that the needle passes through the costoclavicular ligament that runs between the clavicle and the first rib, as that fibrous tissue is dense and hard to dilate later.

\section{ANESTHETIZING THE INSERTION SITE}

A wheal of lidocaine should be placed intradermally at the selected site. Remember that the subcutaneous tissue has no sensory fibers, so that tissue needs no anesthetic. Though the periosteum of the clavicle is quite sensitive, one should not attempt to anesthetize it, as it's hard to do effectively and may compress the vein. Furthermore, as noted, the goal should be to avoid hitting the clavicle at all.

\section{PLACING THE NEEDLE ON THE SYRINGE}

The needle should be placed on the syringe so that the bevel of the needle aligns with the numbers on the $10 \mathrm{cc}$ syringe. This alignment allows the operator to keep the bevel always pointed down towards the feet. This orientation of the needle will help prevent an inadvertent placement of the wire into the internal jugular vein by 'directing' the wire inferiorly, toward the heart. Once the needle is placed on the syringe, draw up 1cc of sterile saline flush into the syringe. Having this saline in the syringe will enhance your ability to see the venous blood flash into the syringe when you enter the vein. Furthermore, when that venous blood touches the saline, rather than dry metal and plastic, the chance that the blood will clot within the syringe is minimized. The saline can also provide a bit of lubrication to the plunger of the syringe.

\section{INSERTING AND AIMING THE NEEDLE}

\section{My aim is true. \\ -Elvis Costello}

Just prior to inserting the needle, some practitioners will stop the ventilator, if the patient is intubated, or even disconnect the endotracheal tube momentarily. The needle is inserted, always visualizing the target site in your mind's eye. Sometimes using the quip 'think like a needle' can be a useful admonition. Again, you should not start the insertion of the needle too far medially as the needle might then pass through the costoclavicular ligament, which can be hard to dilate later to allow the easy passage of the actual catheter. It is also best to avoid 'walking down the clavicle' with your needle, for several reasons. First, hitting the periosteum of the clavicle with your needle can be quite painful for the patient. Second, doing so may clog the needle with tissue, which will impair one's ability to discern accurately when the vein is entered. Also, aiming for the clavicle, instead of the vein, will require distortion of the needle in a manner that will make it more difficult to guide it to the optimal site to enter the vein. This trajectory could also increase the risk of kinking of the guidewire during subsequent dilatation, which will make the eventual passage of the catheter over the wire difficult.

You should strive to 'feel' the tip of the needle as it is inserted, always trying to avoid pushing against or through tough tissue. Your goal should be to have the needle traversing soft tissue while avoiding the tougher tissue of the costoclavicular ligament or the periosteum of the clavicle.

Therefore, the optimal trajectory is a single straight pass of the needle from the insertion site within the lidocaine skin wheal, with the needle passing under the clavicle, towards a point immediately below the sternal notch while visualizing 'the box' sized area of the vein described earlier. The left hand should be positioned with the index finger in the sternal notch and the thumb immediately lateral to the insertion site, with the thumb providing pressure straight down on the soft tissue to help ensure a flat trajectory for the needle.

\section{ENTERING THE VEIN}

Suction on the syringe is created once the needle is beneath the skin, so that one can discern when the vein is actually entered. Some practitioners believe that it is optimal to apply this suction at frequent, small intervals rather than attempting to maintain suction throughout the passage of the needle, which can be a bit challenging. Occasionally, the vein will be compressed side to side as the needle passes through it. If one perceives that the needle has gone far enough to reach the target site on the vein, but has not 'struck' blood, the needle should be withdrawn slowly, while applying suction, so that 
the vein will have a chance to 'spring back open', allowing the syringe to fill with blood.

This first pass of the needle, towards a point directly below the sternal notch where you envision the vein crossing the first rib posterior to the clavicle, should proceed straight and steady until the hub of the needle is buried in the skin. It is not unusual for this first pass of the needle to be 'dry' (that is, no flash of blood is seen). As described above, the needle should be pulled back slowly and steadily, looking again for a flash of blood, until the needle is completely out of the skin. You should understand that there will not always be a flash of blood on your first pass of the needle. If no blood enters the syringe, flush out the saline in the syringe onto a gauze pad, along with any clot or tissue that may have been drawn into the needle and reload the syringe with fresh saline flush. The next pass of the needle should start in a manner identical to the first pass, with the same entrance site, but with a trajectory of the needle tip that is oriented about 10 degrees cranially compared to the first pass. (That is, the tip of the needle is aimed slightly more superiorly.) If subsequent passes are necessary, the needle should again be rotated further cranially.

With each unsuccessful pass of the needle, stop, analyze, and make a conscious decision about where you want to aim next. [Tribble, 2016] Do your best to consciously pass the needle with a slightly different trajectory with each pass. After all, the definition of futility is doing the same thing over and over, expecting a different result. You will surely increase your chance of success, at least by a bit, with careful reassessment after each passage of the needle.

The goal is to see free flow of blood into the syringe. Again, if this free flow of blood doesn't happen as the needle is inserted nor when it is withdrawn, the needle should be taken out of the skin and flushed, to ensure that the lumen of the needle is open and not clogged with blood or tissue. When the needle is reinserted, avoid the temptation to direct it more posteriorly, as this change in direction will endanger the apex of the lung. Prior to reinserting the needle, the landmarks should be reconsidered, carefully. Again, one should continue to orient the bevel of the needle inferiorly so that the wire to be passed once the needle is in the vein will be more likely to be directed inferiorly, towards the brachiocephalic vein, and not superiorly, towards the internal jugular vein.

The greatest risk of hitting the apex of the lung and causing a pneumothorax will be in the thin, elderly patient. Patients who are obese have a slightly lower risk of pneumothorax, though the procedure is made more challenging in these patients by the fact that the needle will have to be inserted deeper, of course. If air is aspirated into the syringe, you will know that you have entered the lung. In this unfortunate circumstance, you should suspend the procedure and obtain a chest $\mathrm{x}$-ray. Thought will need to be given as to what the next steps should be and may include a plan for serial chest $\mathrm{x}$-rays or, possibly, a prophylactic chest drain, especially if the patient is on a ventilator.

What should you do if you continue to be unsuccessful in hitting the vein? Should you let someone else take over? Should you consider other approaches, such as a supraclavicular approach? One principle that should always be adhered to in this setting is to not attempt to cannulate the vein on the opposite side without first obtaining a chest film.

\section{INSERTING THE WIRE WITH THE SELDINGER TECHNIQUE}

When free flow of blood into the syringe is assured, the needle is stabilized carefully. Once again, check the orientation of the needle being sure that the open portion of the beveled tip is 'aimed' inferiorly, as determined by the numbers on the syringe barrel, as noted earlier. Some use a clamp to help with this stabilization. With the needle stabilized, the syringe is removed. Care must be taken to avoid air entrainment into the needle. Covering the needle with the thumb can be part of this process, although this maneuver is probably unnecessary, because the smaller needles in current use have a low risk of the entrainment of air through the needle. However, the most important strategy for avoiding air entrainment is to keep the patient in Trendelenburg and to assure that the patient is not severely hypovolemic, as noted earlier. If the patient is breathing spontaneously, one can also ask the patient to perform a Valsalva maneuver while the needle hub is open, if they are able to cooperate.

One must always consider the possibility that the subclavian artery has been entered. Usually the blood will be redder than expected, or the blood coming out of the needle will be under pressure, and may even be pulsatile, if the needle has entered the artery. The needle can be transduced if there is doubt about which vessel has been entered. If transducing the needle is not feasible, a length of IV tubing can be attached and raised. Blood under venous pressure should not move very far up that tubing as it is held up. That is, the blood should not rise higher than the central venous pressure. However, if the needle is in the artery, the blood will, of course, rise higher in the tubing.

At this point, the wire is inserted into the needle and passed gently into the vein. It's best to orient the J portion of the wire so that the 'open' end of the wire is oriented inferiorly (toward the heart) so that the wire will be most likely to move towards the superior vena cava and not towards the internal jugular vein. Maintain control of the outer end of the wire to keep it sterile and to avoid allowing too much of the wire to be inside the vein. That is, enough wire should be left outside the skin to allow the use of a dilator. If arrhythmias are noted, the wire has entered the heart and should be pulled back a bit. It is worth noting that a minor arrhythmia at this point is actually a sign that the wire is in the correct place, though it should still be pulled back somewhat. Some practitioners advocate getting a chest film when the wire is initially placed to confirm its position, but most do not utilize this strategy.

An inability to advance the wire, despite the needle clearly being inside the vein (with blood easily able to be withdrawn), is a not infrequent and always frustrating experience. It will happen. Perhaps the most important advice to the operator at this point is to slow down and be patient. Achieving a "Zen" state at this point is essential. Getting impatient and 
rushing is a recipe for failure. Often patiently and gently advancing and retracting the wire multiple times, until the patient's breathing opens the vein up just enough to allow the wire to advance, is all that is needed. Sometimes the operator will have to remove the needle and access the vein again in a slightly different trajectory. Some experienced practitioners will turn the wire around and use the "straight" rather than the ' $\mathrm{J}$ ' end of the wire if the needle is in the vein but the wire cannot be advanced. The ' $\mathrm{J}$ ' end can sometimes be an impediment to advancing the wire and that issue can be resolved with this maneuver.

\section{DILATING THE TRACK \& INSERTING THE CATHETER}

The skin at the entry site should be nicked with an 11 blade to keep the dilator from 'hanging up' on the skin. It is important to be sure that the blade has been inserted far enough to cut through the dermis. Make certain that there is no skin 'bridge' between the cut and the wire, which requires considerable attention and may require turning the blade of the knife back towards the wire. The dilator is then placed over the wire and slid carefully into the vein, while rotating the dilator. Very gentle backwards traction is applied to the wire, in order to provide a 'post' or a 'rail' over which the dilator can more readily advance. The wire should intermittently be moved back and forth just a bit, to ensure that it is not kinking, as the dilator is being inserted. The goal is to avoid, assiduously, kinking the wire, as a kink can impede the eventual placement of the catheter over it.

The wire must always be controlled, on one side of the dilator or the other, to avoid 'losing the wire' in the vein, which is easier than one might imagine. The dilator need go no deeper than needed for the tip to enter the vein. Pushing it deeper risks injury to the vein. With each slight push of the dilator into the tract, the wire should be moved back and forth a bit, to ensure that the wire is not being bent. A kink in the wire can make subsequent maneuvers with the dilator or the catheter quite difficult.

Here are diagrams of the steps in the Seldinger technique:

1. The wire is threaded into the needle, once it is in the vein:

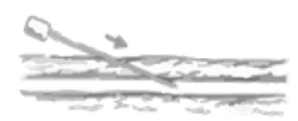

2. The skin is nicked so that the dilator can be threaded over the wire:

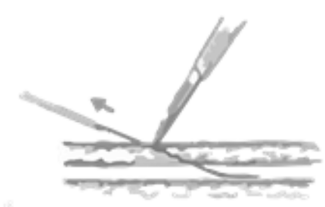

3. The dilator is threaded gently over the wire and withdrawn:

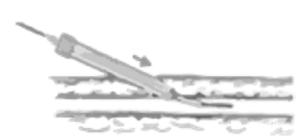

4. The catheter is then inserted, and the wire is withdrawn:

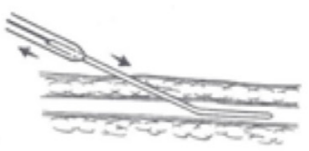

Once the tract is dilated, the catheter can be threaded over the wire.

Here is what a kinked guidewire may look like:

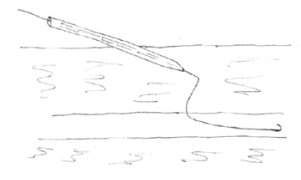

It is apparent that the dilator should not be advanced further on a kinked wire such as the one shown here, as doing so will only accentuate the kink. Obviously, one must attempt to avoid kinking the wire, using the techniques described.

The catheter itself must be preflushed with saline, ensuring that all ports of the catheter are adequately flushed, prior to inserting it over the wire. One must focus on holding the wire to maintain control over it at all times. Once the wire has been threaded into place, the needle is removed and 'stored' safely. The external end of the wire should then be threaded into the most distal port of the line. As noted, all the ports should be flushed and the extra port or ports capped prior to inserting the catheter over the wire. When the catheter has been inserted so that $15 \mathrm{~cm}$ of it is beneath the skin for a right sided line or 18 to $20 \mathrm{~cm}$ for a left sided line, the wire is removed.

\section{CONFIRMING PROPER PLACEMENT OF THE} CATHETER

First of all, the catheter can be aspirated to ensure free flow of blood. The blood aspirated should be inspected again to be sure it does not appear to be arterial. Remember that if the patient is hypotensive there may not be pulsatile flow, even if the catheter is in an artery. And, if the patient is hypoxic, the blood may be dark, even if it is arterial. All lumens of the catheter should be aspirated and then flushed again. A chest $\mathrm{X}$-ray must be obtained to confirm the proper position of the catheter and the absence of a pneumothorax (or a hemothorax). The proper position of the catheter is in the distal superior vena cava. The person who inserted the catheter should be the one who views the chest $\mathrm{X}$-ray. 


\section{STRING THEORY: SECURING THE CATHETER [GREENE, 2010]}

Before placing a dressing, the catheter must be secured to the skin of the chest with at least one stitch placed in the skin. This stitch should be placed so that it can be tied to an eyelet on the catheter, if there is one, or around the catheter if no eyelet is present. The first step in placing such a stitch is to ascertain where the suture should be located so that it is directly beneath the expected attachment site on the catheter. Next, you should consider whether the optimal site for the skin suture or sutures was actually anesthetized earlier in the procedure. It is likely that it was not, so it is often worthwhile to add some additional lidocaine to the precise site you intend to place the suture. You should always insert the needle for additional anesthetic through the previously anesthetized skin. The suture is placed and then tied. However, you should not tie the suture so tightly that it will cause skin necrosis. Some practitioners place a clamp beneath the knot to prevent overtightening against the skin. At least three knots should be used if the suture is braided while six knots should be used if the suture is monofilament. The suture is then tied to, or around, the catheter, in essence securing the catheter between the two sets of knots.

\section{DRESSING THE CATHETER}

Placing a dressing over the catheter while the sterile field is still in place is essential. The skin around the catheter will never be as clean as it is at that point, and if a dressing is put in place with a good skin adhesive it should stay sterile for days. The protocol includes the use of an antibiotic or antiseptic impregnated disc placed over or around the entry site of the catheter, which is then covered by a clear plastic dressing. A "vest-over-pants" catheter dressing has become popular as it helps seal the dressing around the catheter hub.

The following image shows such a dressing, with the main part of the dressing being 'the pants' and with the secondary part being 'the vest' that overlaps 'the pants,' locking the hub of the catheter in place and completing the dressing. Here is what such a dressing looks like:

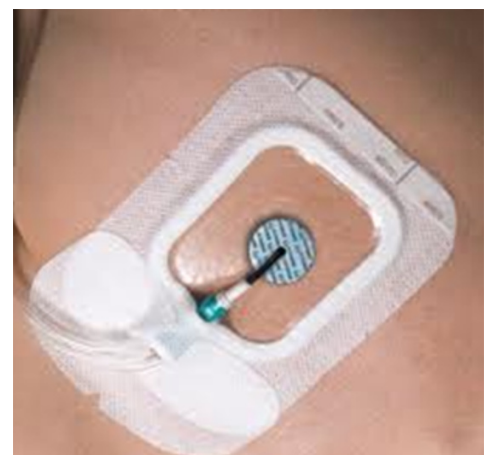

The skin around the catheter should be liberally coated with benzoin solution or some other sticky, skin protective solution prior to placing the dressing over the catheter. Doing so insures that the catheter dressing will adhere tightly and will help to minimize the chance of infection. Considerable attention should be paid to this dressing, no matter what the catheter is to be used for. The risk of infection is considerable but that risk is even greater if the line is to be used for parenteral alimentation.

\section{CLEANING UP AFTER YOURSELF}

You should properly dispose of all 'sharps' (needles and blades) in an appropriate container. Many kits have materials in which to stick the sharps as they are used to minimize the risks of injury to team members. The more you clean up after yourself, the safer the environment will be and the more popular you will be with your nursing colleagues.

\section{NO JOB IS FINISHED UNTIL THE PAPERWORK IS DONE!}

A procedure note should be always be entered into the chart or electronic medical record (EMR), as soon as feasible after completing the procedure. And, you should eventually write down any reflections or lessons learned during the procedure in a Moleskine notebook (as you should do after virtually all procedures). [Tribble, Merrill 2016]

\section{SUMMARY}

Experience is communicated by small details intimately observed. —Ernest Hemingway

Placing percutaneous central lines in the subclavian vein can be complicated and is associated with significant risks. Detailed knowledge of the anatomy of the subclavian vein is essential. Understanding each of the steps in this process is crucial. However, with proper preparation and careful attention to detail, subclavian lines can be placed efficiently, accurately, and safely.

\section{LAGNIAPPE}

Finally, we would like to suggest a song one might hum when placing a central line, following the well-known adage that humming can be calming:

I am a lineman for the county,

And I drive the main roads.

Searching in the sun for another overload.

I bear you singing in the wires,

I can bear you through the whine.

And the Wichita lineman,

Is still on the line.

-Wichita Lineman, Glen Campbell, Michelle Shocked and The Texas Tornados (Composer: Jimmy Webb, 1968) 


\section{REFERENCES}

Buffett,W.https://www.brainyquote.com/quotes/warren_buffett_138173 \#: :text=Risk\%20comes\%20from\%20not\%20knowing\%20what\%20 you're\%20doing (Accessed August 5, 2021)

Hester D. 2014. An Intern's Recollection of a Night at the VA, July 2004. Chest 146(3): 871

Dudrick S. Steiger E, Wilmore D. 1969. Continuous Long-term Venous Catheterization: An Adjunct to Surgical Care and Study. Curr Top Surg Res. 1: 325-40.

Dudrick S. 2003. Early Development and Clinical Application of Total Parenteral Nutrition. J Parenteral and Enteral Nutrition. 27 (4): 291-9.

Patrick S, Tijunelis M, Johnson S, Herbert M. 2009. Supraclavicular Subclavian Vein Catheterization: The Forgotten Central Line. WestJEM 10: $110-4$.

Honore P, Redant S, Preseau T. 2021. Use of peripherally inserted central catheters in ICU patients: We need to put in balance the important risks versus the potential advantages. J Crit Care. In Press.

Tubbs R. 2021. Anatomy is Destiny. Clinical Anatomy 34: 325.

Gretsky W. https://www.brainyquote.com/authors/wayne-gretzky-quotes (Accessed August 5, 2021)

Tomar G, Chawla S, Ganguly S, Cherian G, Tiwari A. 2013. Supraclavicular approach of central venous catheter insertion in critical patients in emergency settings: Re-visited. Indian J Crit Care Med. 17(1): 10-15.

Tribble C, Julliard W. 2019. First, We Do Harm: Obtaining Informed Consent for Surgical Procedures. The Heart Surgery Forum 22(5): E423-8.

Goldratt, Eliyahu M. 1994. It's Not Luck.

The North River Press. Great Barrington, MA (A novel written to explain The Theory of Constraints)

Tribble C. 2020. The Mental Strategies of Surgeons: A Primer, Part I. Heart Surgery Forum 23(6): 927-36.

Tribble C. 2021. The Mental Strategies of Surgeons: A Primer, Part II. Heart Surgery Forum 24(1): 201-208.

Waters D. 1995. A Heart Surgeon's Little Instruction Book. Quality Medical Publishing, Inc. St. Louis, Missouri.

Video of donning gloves after gowning: http://www.brooksidepress.org/ Products/Scrub_Gown_and_Glove_Procedures/lesson_1_Section_6.htm

Mischke C, Verbeek J, Saarto A, Lavoie M, Pahwa M, Ijaz S. 2014. Gloves, extra gloves or special types of gloves for preventing percutaneous injuries in healthcare personnel. Cochrane Database Syst Rev. 7(3): CD009573.

Calland J, Guerlain S, Adams R, Tribble C, Foley E, Chekan E. 2002. A Systems Approach to Surgical Safety. Surg Endosc 16: 1005 - 14.

McPhee J. 1999. A Sense of Where You Are: A Profile of Bill Bradley at Princeton. Farrar, Straus and Giroux. New York.

Costello E. 1977. Allison. https://genius.com > Elvis-costello-alison-lyrics (Accessed August 5, 2021)
Tribble C. 2016. A Practical Minded Obsession with the Possibility and Consequence of Failure. Heart Surgery Forum Journal 19(1): 1-4.

Greene B. 2010. The Elegant Universe: Superstrings, Hidden Dimensions, and the Quest for the Ultimate Theory. W. W. Norton \& Company. New York.

Tribble C, Merrill W. 2016. In Your Own Words: Toward a More Perfect Union of Patient Care and Education. Ann Thorac Surg 101: 837-40.

Hemingway E. https://writewisdom.com/carousel/ernest-hemingway (Accessed August 5, 2021)

Wichita Lineman. https://www.youtube.com/watch?v=Q8P_xTBpAcY (Accessed August 5, 2021)

\section{ADDITIONAL REFERENCES}

Brandon O. 2017. 8 Advanced Tricks for Central Line Placement. Critical Concepts: Meditations on Critical Illness. www.critcon/archives/294

Sanchez J, Daly J. 2010. Stanley Dudrick, MD: A Paradigm Shift. Arch Surg 145 (6): 512-4.

Tribble C. 2016. Failing to Prepare is Preparing to Fail. Heart Surgery Forum Journal 19: 112-5.

Czarnik T, Gawda R, Perkowski T, Weron R. 2009. Supraclavicular Approach is an Easy and Safe Method of Subclavian Vein Catheterization, Even in Mechanically Ventilated Patients. Anesthesiology 111 (2): 334-9

Yoffa D. 1965. Supraclavicular Subclavian Venepuncture and Catheterisation. The Lancet 2: 614-7.

Grant J. 1980. Handbook of Total Parenteral Nutrition. WB Saunders Company. Philadelphia.

Link to video of subclavian line insertion on the New England Journal of Medicine web site: http://www.nejm.org/doi/full/10.1056/ NEJMvcm074357

\section{THE AUTHORS}

The authors of this treatise include three Thoracic Surgeons (NT, RT, CT) and three surgeons with Surgical Critical Care Certificates (CH, NT, CT). Two are faculty surgeons at The University of Virginia (NT, CT), and all four of the authors received significant portions of their training at The University of Virginia. All of the authors have dedicated significant portions of their careers to medical and surgical education.

\section{ACKNOWLEDGEMENTS}

Ben Tribble and Jake Van Yahres helped create the artwork for this paper. 